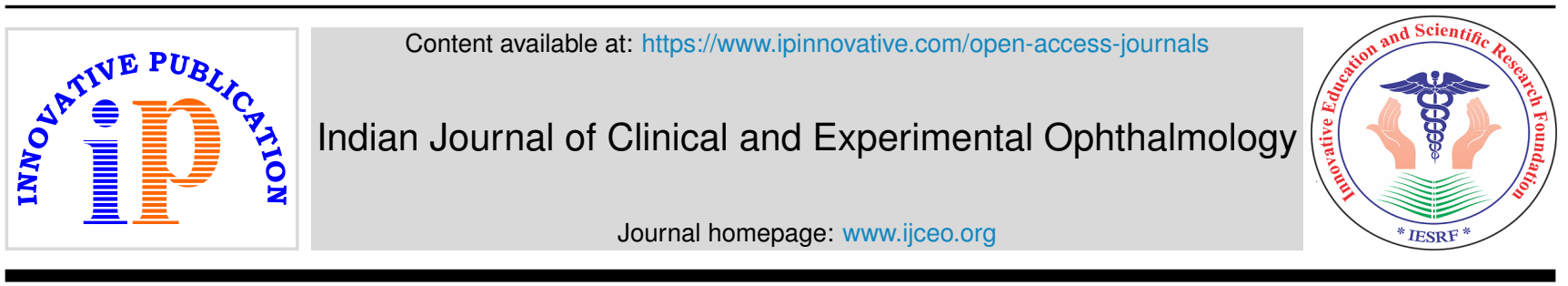

Original Research Article

\title{
Study of prevalence of raised IOP in post cataract patients following topical steroid usage
}

\author{
Kavitha Mohankumar ${ }^{1, *}$, Abhay Amrit Lune ${ }^{1}$, Rakesh Goud ${ }^{1}$, \\ Chaitali Chandrashekhar Desai ${ }^{1}$, Namratha Judith Cardoza ${ }^{1}$ \\ ${ }^{1}$ Dept. of Ophthalmology, Dr. D.Y Patil Medical College \& Research Centre, Pune, Maharashtra, India
}

\section{A R T I C L E I N F O}

\section{Article history:}

Received 02-12-2020

Accepted 26-12-2020

Available online 30-06-2021

\section{Keywords:}

Steroids

Intra-ocular pressure

\begin{abstract}
A B S T R A C T
Purpose: To evaluate the rise in intraocular pressure (IOP) in post cataract surgery patients who received topical steroids.

Materials and Methods: Patients in the age group of 50-75 years and both sexes who were coming to ophthalmology OPD at a tertiary care hospital of Western Maharashtra were studied. 500 subjects who underwent uncomplicated cataract surgery were taken into consideration; both age and sex matched. All patients were screened for raised intraocular pressure by Perkin's tonometer preoperatively and at 5th day, 2nd week, 4th week, 6th week and 8th week postoperatively. Patients were allocated into two groups: either topical Prednisolone acetate e/d (1\%) (Group I) or Dexamethasone e/d (0.1\%) (Group II) started six times for 1 st week (considering the cells in anterior chamber $<2+$ ) \& then tapered off subsequently in the following weeks for a period of 5 weeks (starting with six times for 1st week, then tapering to 4 times for the next week, 3 times for the next week, 2 times for the next week, 1 time for the next week and then stopped).

Results: In our study group, the prevalence of steroid-responders was 3.6\% with $0.8 \%$ showing mild rise, $2.8 \%$ showing moderate rise in IOP on the fourth week of topical steroid usage. There was no difference in the mean IOP rise with the usage of two different topical steroids (E/d Prednisolone acetate (1\%) and E/d Dexamethasone $(0.1 \%)$ ) in our study. The highest mean IOP was noted at the 5 th postoperative day (20.26 $\mathrm{mmHg}$ ) which was due to inflammation \& there was a gradual decrease in IOP in the subsequent weeks of follow-up till 8th week. While as duration after cataract surgery progress till 8th week of follow up, moderate rise in IOP occurs which gradually comes down to mild rise till 8 th week of follow-up.

Conclusion: Steroid-induced IOP elevation typically occurs after 4 weeks of beginning steroid therapy and the prevalence of steroid responders in post cataract surgery patients was established.
\end{abstract}

(C) This is an open access article distributed under the terms of the Creative Commons Attribution License (https://creativecommons.org/licenses/by/4.0/) which permits unrestricted use, distribution, and reproduction in any medium, provided the original author and source are credited.

\section{Introduction}

Corticosteroids are a group of anti-inflammatory drugs that are commonly used to treat numerous ocular and general systemic conditions; particularly within the ophthalmic post-operative setting. ${ }^{1-3}$ They act by inhibiting the processes concerned at the location of tissue injury including edema, vasodilation, fibrin deposition, leukocyte migration, capillary and fibroblast proliferation, collagen

\footnotetext{
* Corresponding author.

E-mail address: kavithamhn123@gmail.com (K. Mohankumar).
}

deposition and scar formation.

In the mechanism of action, induction of lipocortins play a significant role as these lipocortins inhibit phospholipase A2. Phospholipase A2 ends up in arachidonic acid production from inflammed cells that inturn stimulates the assembly of prostaglandins \& leukotrienes.

Mucopolysaccharides accumulate within the aqueous outflow pathways, inflicting resistance with inhibition of prostaglandins $\mathrm{E} \& \mathrm{~F}$ that typically lower the IOP by increasing the outflow facility \& reduced phagocytosis of debris by the endothelial cells from trabecular meshwork. 
The use of corticosteroids may lead to important ocular side effects. Among the ocular side effects, cataract and glaucoma are common.

IOP elevation following steroid use is well-documented. Steroids are well-known to induce ocular hypertension when administered with topical, periocular, and even general systemic or inhalational routes Some people are well- known to experience a high degree of IOP elevation with low doses or short durations of treatment with topical corticosteroids. Around one third of individuals experience moderate increase in IOP following topical steroid use. But $5-6 \%$ of normal population will develop an increase in IOP after 4 weeks of topical steroid therapy. ${ }^{4-7}$ Thus $5 \%$ of the overall general population is taken into consideration to be "high steroid responder", i.e., may develop steroid induced rise in IOP when steroids are administered. Topical corticosteroids are usually used following cataract surgery to decrease the inflammation and improve the visual outcomes. Steroids inhibit the production of leukotrienes $\&$ prostaglandins post surgery, thereby reducing ocular inflammation. Though steroids facilitate in decreasing inflammation by speeding up recovery and improving visual outcomes, they are well-known to have important side effects, whether topically or systemically administered.

Steroid response is defined as an IOP increase greater than $25 \%$ from baseline value while on topical steroids used 4 times daily for a period of 4 weeks followed by a decrease of more than $25 \%$ after topical steroids was discontinued for a period of 2 weeks. Considering the studies done by Armaly et al., low medium \& high steroid responders are those who develop rise in IOP of $<6 \mathrm{mmHg}, 6-15 \mathrm{mmHg}$ and $>15 \mathrm{mmHg}$ within a period of 4 weeks with the usage of topical steroids starting four times daily.

While the mechanism of steroid response is not yet elucidated, it has been calculated that $5 \%$ of general population might be classified as high steroid responders \& therefore might develop a considerably raised IOP of over $15 \mathrm{mmHg}$ from the baseline on exposure to topical steroids.

General population response to steroids can be graded as non-responders, responders and super responders. According to the study done by Becker in 1965, all primary open-angle glaucoma patients elucidated IOP rise with administration of topical Betamethasone $(0.1 \%)$ for four weeks. Among normal individuals also two varieties of responses were observed, $70 \%$ showed small rise of IOP while 30\% showed high pressure response to topical steroid administration for four weeks. ${ }^{4,8}$ Once the steroids were withdrawn, most of the eyes returned to the baseline values within three weeks.

It has been reported that the elevation of IOP resulting after systemic steroid administration is much less as compared to topical steroids. IOP elevations are more prominent in older adults \& in children when compared with younger adults.
The efficacy of topical corticosteroids as ocular anti-inflammatory drugs following cataract surgery is well-documented. They also help to prevent a range of complications associated with post-operative ocular inflammation, as well as corneal edema \& cystoid macular edema.

Hence our study is conducted with an intention to seek out the prevalence of steroid-induced rise in IOP in post cataract surgery patients. In addition, the study can decipher the comparison of rise in IOP on administration of 2 completely different topical steroid preparations and on adopting 2 different techniques of cataract extraction.

\section{Materials and Methods}

A hospital based longitudinal prospective study was conducted in a tertiary hospital and research care centre in Pimpri, Pune from the period of September 2018 to August 2020 after clearance from the Ethics committee of the institute. Informed consent was taken from all the subjects. The study was conducted in a total of 500 subjects in the age group of 50-75 years including both the sexes who underwent routine, uncomplicated cataract surgery. The inclusion criteria was all consecutive patients in the age group of 50 - 75 years, i.e., senile cataract undergoing routine uncomplicated cataract surgery who receive either topical Prednisolone acetate e/d or Dexamethasone e/d for a period of 5 weeks post-operatively and with cells $<2$ + in anterior chamber (on slit lamp examination). The exclusion criteria were complicated cataract, paediatric cataract, traumatic cataract, any previous ocular surgery, cataract surgery with intraoperative complications, postoperative intense inflammation/ infection, patients on systemic steroid therapy for co-existing systemic disease, patients who have received previous intravitreal/ subtenon's steroids, patients on preoperative steroid treatment (topical and/or systemic), patients on systemic steroid treatment postoperatively, any ocular condition requiring prolonged steroid use (4-6 weeks), known patient of glaucoma / already on antiglaucoma medication, pigment dispersion syndrome, pseudoexfoliation syndrome, connective tissue disorders and type I Diabetes Mellitus.

Participant Information Sheet(PIS) regarding the details of the study were prepared in English, Marathi and Hindi languages. PIS was given to the participants and they were explained about the type and purpose of the study according to the language best understood. After well informed consent, they were enrolled in the study.

Patient's rights for participation in the study were safeguarded. Participation in the study was voluntary. Participants were free to withdraw from the study at any point without giving any reason and without any loss to medical care.

Brief history was taken along with important demographic factors. Detailed history of other co-morbid 
conditions was taken. All the participants have undergone a thorough ophthalmologic examination.

Vision including best corrected visual acuity was assessed using illuminated Snellen's chart. Detailed anterior segment evaluation was done along with fundus evaluation using slit lamp biomicroscopy/ indirect ophthalmoscopy. Intraocular pressure assessment with Perkin's Tonometer was carried out preoperatively and at weeks - first week $\left(5^{\text {th }}\right.$ day), second week ( $14^{\text {th }}$ day), fourth week, sixth week, \& eighth weeks postoperatively. The average of two separate measurements was recorded. In cases where there was a difference greater than $5 \mathrm{~mm} \mathrm{Hg}$ (millimeters of mercury), a third measurement was taken. The average of the two closest IOP measurements was then used for the analysis. Fundus examination was also done preoperatively, at 2 weeks, 4 weeks, 6 weeks, \& at 8 weeks.

Patients were divided into 2 groups based on their exposure to either topical Prednisolone acetate e/d (1\%) (Group I) or Dexamethasone e/d (0.1\%) (Group II) started six times for $1^{\text {st }}$ week (considering the cells in anterior chamber $<2+$ ) \& then tapered off subsequently in the following weeks for a period of 5 weeks.

The following parameters were assessed in each group and their significance verified by statistical means:

1. Increase in IOP from baseline.

2. Classification into mild, moderate \& severe groups.

3. Difference in rise in IOP with usage of $\mathrm{E} / \mathrm{d}$ Prednisolone $(1 \%)$ \& with E/d Dexamethasone $(0.1 \%)$.

4. Difference in rise in IOP with different time periods

5. Age \& Sex.

Steroid response will be graded according to the rise in IOP from baseline as done by Armaly et al. after applying topical steroids for a period of 5 weeks, starting with 6 times per day (considering the cells in anterior chamber $<2+$ ) \& tapering the steroid dose subsequently each week:-

Low: rise in IOP $<6 \mathrm{~mm} \mathrm{Hg}$

Medium: rise in IOP of 6-15 mm Hg

High: rise in IOP $>15 \mathrm{~mm} \mathrm{Hg}$

The mean IOP rise from baseline was analysed and classified either as Steroid Responder or not a Steroid Responder and according to the severity of steroid response, if noted, in each of the groups. Results and conclusion were drawn from the same.

All details of participants were kept under strict confidentiality. Microsoft excel was used to enter data and statistical analysis was done using software SPSS version 2.0. Analysis of quantitative data was done in terms of Mean and Standard deviation. Analysis of qualitative data was done using appropriate test of significance like t-test.

\section{Results}

According to our study, steroid-induced IOP elevation following cataract surgery was found to occur after four weeks of beginning topical steroid therapy. The prevalence of steroid-responders in our study was $3.6 \%$ with $0.8 \%$ showing mild rise, $2.8 \%$ showing moderate rise and no subjects showing severe rise in IOP on the fourth week of topical steroid usage. There was no difference in the mean IOP rise with the usage of two different topical steroids (E/d Prednisolone acetate $(1 \%)$ and E/d Dexamethasone $(0.1 \%))$ or with the application of two different techniques (SICS and Phacoemulsification) in our study. There was no difference between the mean IOP of subjects with or without any comorbidities. The highest mean IOP was noted at the 5 th postoperative day $(20.26 \mathrm{mmHg})$ which was due to inflammation \& there was a gradual decrease in IOP in the subsequent weeks of follow-up till 8th week. While as duration after cataract surgery progress till 8th week of follow up, moderate rise in IOP occurs which gradually comes down to mild rise till $8^{\text {th }}$ week of follow-up.

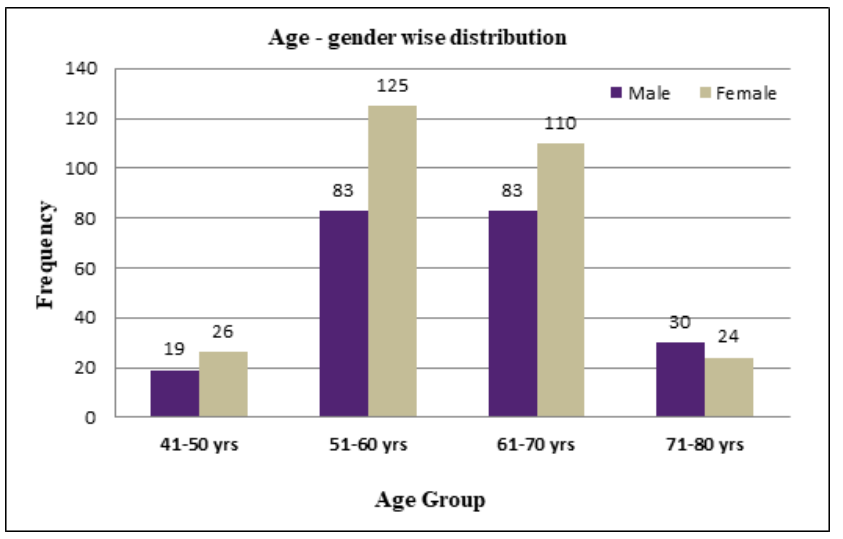

Fig. 1: Showing age and gender wise distribution of study sample

Table 1:

$\begin{array}{lc}\text { Age (years) Statistics } & \\ & 500 \\ \text { Mean } & 61.37 \\ \text { Std. Error of Mean } & .323 \\ \text { Std. Deviation } & 7.22 \\ \text { Range } & 26 \\ \text { Minimum } & 50 \\ \text { Maximum } & 75\end{array}$

\section{Discussion}

It was found that the mean age for 500 study sample size was 61.37 years with standard deviation of 7.22 years, with the highest 75 years and lowest 50 years. There were $285(57 \%)$ females and 215 (43\%) males in the study. 208 (41.6\%) subjects were from 51-60 years age group followed by 193 $(38.6 \%)$ subjects in 61-70 years age group and 99 (19.8\%) subjects in 71-75 years age group. For this study, the range of ages that were considered were $50-75$ years. 
Table 2: IOP (mm Hg) estimates at different time interval

\begin{tabular}{lcccc}
\hline Time & Mean & Std. Error & \multicolumn{2}{c}{ 95\% Confidence Interval } \\
Upper Bound \\
Pre-operative & 14.776 & .110 & 14.560 & 14.992 \\
5th Day & 24.222 & .129 & 23.968 & 24.476 \\
2nd Week & 23.024 & .127 & 22.775 & 23.273 \\
4th Week & 12.672 & .161 & 12.356 & 12.988 \\
6th Week & 13.192 & .112 & 12.973 & 13.411 \\
8th Week & 12.564 & .109 & 12.350 & 12.778 \\
\hline
\end{tabular}

Table 3:

\begin{tabular}{lcccccc}
\hline Time duration & Value & F & Hypothesis df & Error df & $\begin{array}{c}\text { Sig. } \\
\text { Partial Eta } \\
\text { Squared }\end{array}$ & $\begin{array}{c}\text {. } \\
\text { Pillai's Trace }\end{array}$ \\
Wilks' Lambda & .975 & $3.824 \mathrm{E} 3 \mathrm{a}$ & 5.000 & 495.000 & .000 & .975 \\
Hotelling's Trace & .025 & $3.824 \mathrm{E} 3 \mathrm{a}$ & 5.000 & 495.000 & .000 & .975 \\
Roy's Largest Root & 38.625 & $3.824 \mathrm{E} 3 \mathrm{a}$ & 5.000 & 495.000 & .000 & .975 \\
\hline
\end{tabular}

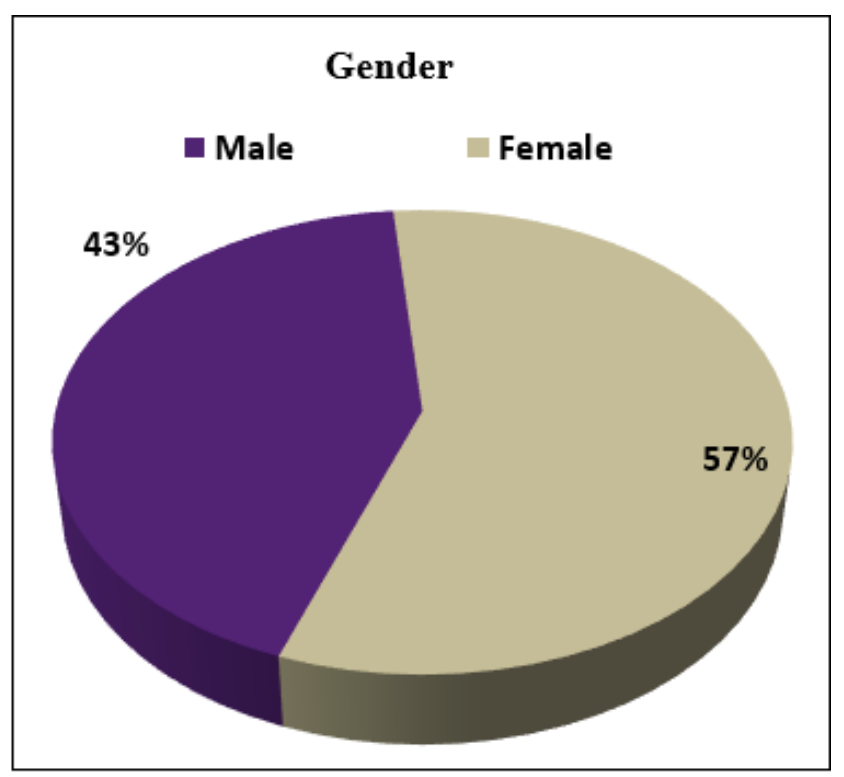

Fig. 2:

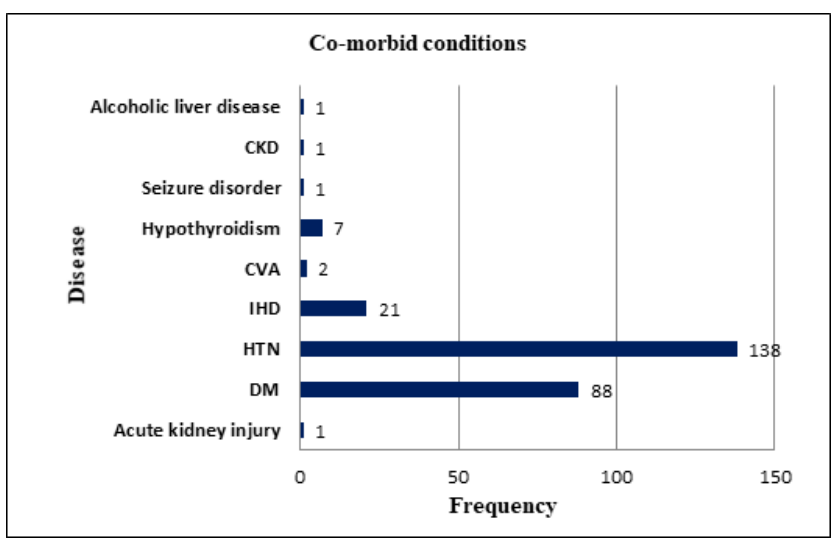

Fig. 3: Showing comorbid conditions among study subjects

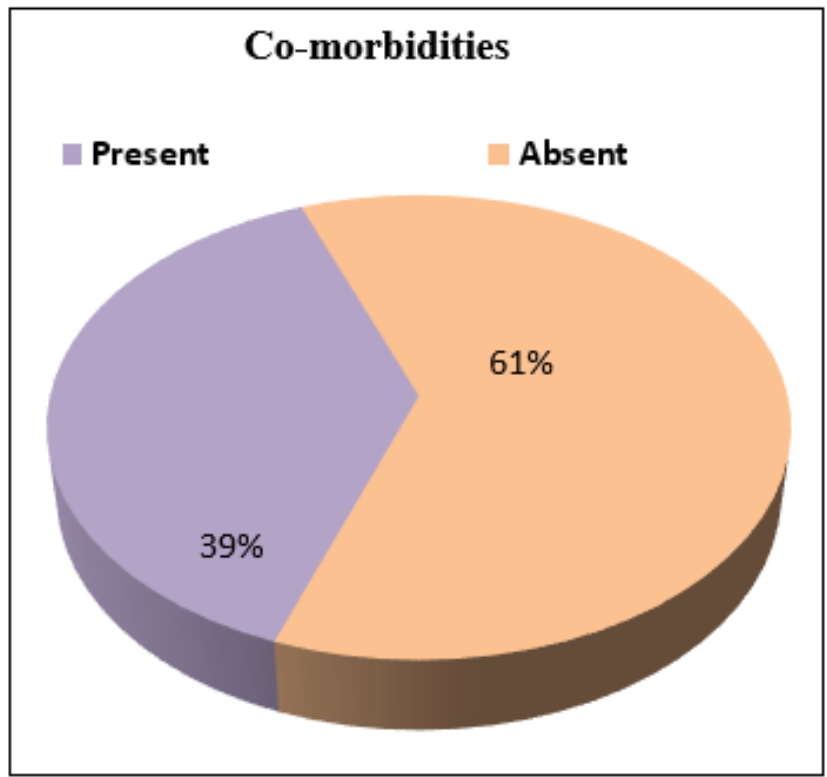

Fig. 4: Showing prevalence of comorbidities among study subjects

The study by Armaly demonstrated that older adult patients were at higher risk for steroid-induced increase in IOP postoperatively than younger adult patients. ${ }^{5} \mathrm{~A}$ study was conducted by Kusne Y et al., in which 3488 eyes of 3488 patients were included. A retrospective analysis was done regarding the post-operative IOP rise after cataract surgery following the usage of topical steroids and they did not find any statistically significant difference between the age group and the genders as compared to the IOP rise after surgery. ${ }^{9}$

These findings were comparable to our study which enrolled 500 patients with a mean age of 61.37 years. There were $285(57 \%)$ females and $215(43 \%)$ males in our study. We also did not find any statistically significant difference 
between age group and gender diversification and steroidinduced post-operative IOP rise.

Sathyan $\mathrm{P}$ et al., conducted a study in which they enrolled 2634 patients aged between 26-90 years. A three year prospective analysis was done. Systemic comorbidities were present in $51.29 \%$ of patients, while $38.19 \%$ patients having more than one systemic comorbidity. Diabetes mellitus $(n=813,60.18 \%)$ and hypertension $(n=840,62.18 \%)$ were reported to be the commonest comorbidity along with cardiac disorders $(n=203)$, bronchial asthma $(n=60)$, thyroid disorders $(n=34)$, renal diseases $(n=1)$ and HIV + status $(\mathrm{n}=2) .10$

These findings were similar to our study in which 193 patients had systemic co-morbidities. Hypertension (27.6\%) was most common co-morbid condition among study sample followed by diabetes mellitus (17.6\%). Ischemic heart disease, hypothyroidism, kidney diseases, etc., were also present in few cases, some subjects were having more than one comorbid conditions like diabetes with hypertension etc. All total 193 subjects having comorbidities were under oral drugs treatment for the same.

Dodhiya K et al., enrolled 500 patients in their study and followed the patients upto six weeks to observe the IOP changes after cataract surgery following steroid use. They observed that 105 patients $(21 \%)$ had an IOP rise of more than $6 \mathrm{~mm}$ of $\mathrm{Hg}$ of which $15(3 \%)$ had more than $15 \mathrm{~mm}$ of $\mathrm{Hg}$ after six weeks of cataract surgery. At the end of 6 weeks of cataract surgery, $75 \%$ (375) of the patients had an IOP of less than $20 \mathrm{~mm}$ of $\mathrm{Hg}$ and were classified as low steroid responder, $23 \%$ (115) had between 20-30 mm of $\mathrm{Hg}$ which were classified as intermediate steroid responder, and $2 \%$ (10) had IOP of more than $30 \mathrm{~mm}$ of $\mathrm{Hg}$ which were concluded to be high steroid responders. ${ }^{11}$

In our study, 18 subjects were having more than $25 \%$ rise in IOP from baseline value (steroid responders); so prevalence of steroid-responders was $3.6 \%$ among study sample with 4 subjects $(0.8 \%)$ having mild rise and 14 subjects $(2.8 \%)$ with moderate rise in IOP on 4 th week of follow-up.

According to studies conducted by Armaly et al., by applying topical steroids in patients 3 times daily for a period of 4 weeks \& taking IOP change as the parameter, $<6(66 \%)$ were low steroid responders, 6-15 (29\%) were intermediate steroid responders \& $>15$ (5\%) were high steroid responders. ${ }^{4-7}$ According to studies conducted by Becker et al, by applying topical steroids in patients 4 times daily for a period of 6 weeks \& taking final IOP as the parameter, $<20(58 \%)$ were low steroid responders, 20-31 $(36 \%)$ were intermediate steroid responders \& >31 $(6 \%)$ were high steroid responders. ${ }^{4-7}$ It was also observed in our study that $77 \%$ had mild rise \& $23 \%$ had moderate rise in IOP by $4^{t h}$ postoperative week. $86.6 \%$ subjects had mild rise in IOP \& $13.4 \%$ had moderate rise in IOP on $6^{\text {th }}$ postoperative week. $90.8 \%$ had mild rise $\& 9.2 \%$ had moderate rise on 8th postoperative week. There were no subjects with severe rise in IOP during $4^{t h}, 6^{t h}$ and $8^{\text {th }}$ postoperative weeks of follow-up.

$96.8 \%$ of subjects were having moderate rise in IOP (6 - $15 \mathrm{~mm} \mathrm{Hg}$ ) change followed by $3 \%$ subjects with mild rise in IOP $(<6 \mathrm{mmHg})$ and $0.2 \%$ of subjects with severe rise in IOP $(>15 \mathrm{mmHg})$ after cataract surgery on $5^{\text {th }}$ day. While as duration after cataract surgery progress till 8th week of follow-up, there were no subjects with severe rise in IOP; suggesting that after cataract surgery with the usage of topical steroids, moderate rise in IOP occurs which gradually comes down to mild rise till $8^{\text {th }}$ week of postoperative follow-up.

In our study with a similar sample size we observed that there was statistically significant difference $(\mathrm{p}=0.000)$ in mean IOP $(\mathrm{mm} \mathrm{Hg})$ between preoperative with next duration of follow up. The mean IOP on $5^{\text {th }}$ day $(24.22 \mathrm{~mm}$ $\mathrm{Hg}$ ) following cataract was higher than pre-operative (14.77 $\mathrm{mm} \mathrm{Hg}$ ), which gradually decreases till last $8^{\text {th }}$ week follow up. This high IOP immediately after first post-operative day can also sometimes be attributed to retained viscoelastic substances, vitreous loss etc, amongst other causes.

These results are similar to the results found in literature and the cause can be attributed to proportional decrease in steroid dosage at the end of 4-6 weeks.

A study conducted by Matthew $\mathrm{K}$ et al., compared the steroid-induced IOP rise post cataract surgery in 100 patients in which patients were grouped into those receiving prednisolone and dexamethasone eye drops as a treatment for post-operative inflammation. They found a statistically significant difference between IOP rise in dexamethasone group as compared to prednisolone group on thus concluding that dexamethasone eye drops were relatively safer as far as steroid-induced post-operative IOP rise is concerned. ${ }^{12}$

However in our study, we observed 13 out of 18 were receiving Prednisolone eye drops and 5 with Moxi D eye drops with 4 subject having mild rise and 14 with moderate rise in IOP on 4th week follow-up. 174 (34.8\%) subjects were treated with Moxi-D eyedrops while 326 (65.2\%) were treated with Prednisolone eye drops. There was no significant difference between mean IOP of two drugs during any particular time interval. $(\mathrm{p}>0.05)$

Deshpande $\mathrm{S}$ et al., enrolled 101 patients in their study in which they compared changes in steroid-induced postoperative IOP after small incision cataract surgery and after phaco emulsification and did not find any statistical difference between the two modes of surgery. ${ }^{13}$

This is in coherence with our study in which 154 (30.8\%) subjects were treated with phacoemulsification while 346 $(69.2 \%)$ were treated with SICS. There was no significant difference between mean IOP of two types of surgery (SICS $\&$ phacoemulsification) during any particular time interval. $(\mathrm{p}>0.05)$. 
According to studies done by Armaly \& Becker et al., approximately one-third of individuals experience moderate increase in IOP after topical steroid use. However, 5-6\% of normal population develops a marked increase of IOP after 4-6 weeks of topical steroid therapy. ${ }^{4-7}$ Thus, $5 \%$ of the general population is considered to be "steroid responder".

These results were in concordance with our study which showed 18 subjects having more than $25 \%$ rise in IOP from baseline value (steroid responders); so prevalence of steroidresponders was $3.6 \%$ among study sample. 13 out of 18 (2.6\%) were receiving E/d Prednisolone acetate (1\%) and $5(1 \%)$ were receiving E/d Moxi-D $(0.5 \%, 0.1 \%)$ with 4 subjects $(0.8 \%)$ having mild rise and 14 subjects $(2.8 \%)$ with moderate rise in IOP on 4th week follow-up, while $15(3 \%)$ were operated by SICS and $3(0.6 \%)$ by phacoemulsification.

\section{Conclusion}

Steroid-induced IOP elevation typically occurs after 4 weeks of beginning steroid therapy. The prevalence of steroid responders is very less in our study sample, being $3.6 \%$; thus coming to a conclusion that topical steroids can be used in post cataract surgery patients and the rise in IOP noticed in the first few weeks following cataract surgery is mostly due to inflammation. In most cases, the IOP lowers spontaneously to the baseline within 2 weeks upon stopping the steroid.

\section{Source of Funding}

None.

\section{Conflict of Interest}

None.

\section{References}

1. The Loteprednol Etabonate Postoperative Inflammation Study Group 2. A double-masked, placebo-controlled evaluation of $0.5 \%$ loteprednol etabonate in the treatment of postoperative inflammation. The Loteprednol Etabonate Postoperative Inflammation Study Group 2. Ophthalmology. 1998;105:1780-6.

2. Bron A, Denis P, Hoang-Xuan TC, Boureau-Andrieux C, Crozafon P, Hachet E. The Effects of Rimexolone 1\% in Postoperative Inflammation after Cataract Extraction. A Double-Masked Placebo-Controlled Study. Eur J Ophthalmol. 1998;8(1):16-21. doi:10. 177212067219800800105 .
3. Korenfeld MS, Silverstein SM, Cooke DL, Vogel R, Crockett RS. Difluprednate ophthalmic emulsion $0.05 \%$ for postoperative inflammation and pain. J Cataract Refract Surg. 2009;35(1):26-34. doi:10.1016/1.jcrs.2008.09.024

4. Becker B. Intraocular pressure response to topical corticosteroids. Invest Ophthalmol. 1965;4:198-205.

5. Armaly MF. Statistical attributes of the steroid hypertensive response in the clinically normal eye .1:The demonstration of three levels of response. Invest Ophthalmol. 1965;4:187-97.

6. Armaly MF. The Heritable Nature of Dexamethasone-Induced Ocular Hypertension. Arch Ophthalmol. 1966;75(1):32-5. doi:10.1001/archopht.1966.00970050034007.

7. Armaly MF. Inheritance of Dexamethasone Hypertension and Glaucoma. Arch Ophthalmol. 1967;77(6):747-51. d01:10.1001/archopht.1967.00980020749006.

8. Armaly MF. Genetic factors related to glaucoma. Ann N Y Acad Sci. 1968; 151:861-75.

9. Kusne Y, Kang P, Fintelmann R. A retrospective analysis of intraocular pressure changes after cataract surgery with the use of prednisolone acetate $1 \%$ versus difluprednate $0.05 \%$. Clin Ophthalmol. 2016;10:2329-36. do1:10.214//opth.sI21849.

10. Sathyan P. A Three Year Analysis of Systemic Comorbidities in Cataract Operated Patients in India. J Clin Diagn Res. 2017;11(9):3. doi:10.7860/jcdr/2017/30410.10682

11. Dodiya K, Aggrawal S, Bareth K, Shah N. Study of Steroid Induced Rise in Intraocular Pressure Using Non-contact Tonometer After Cataract Surgery in Camp Patients at P.D.U. Medical College Rajkot, Gujarat. Natl J Med Res. 2012;2(2):169-72.

12. Mathew K, Thomas L. Comparison of three different eye drops and assessment of IOP changes in post-operative cataract patients. Int $J$ Med Res. 2018;3(4):31-3.

13. Deshpande S, Israni N, Narayanam S, Bhalla N. A comparative study of effect of SICS and phacoemulsification on corneal endothelium. Indian J Clin Exp Ophthalmol. 2016;2(3):186-9. 101:10.5958/2395ए43 $201600041 x$

\section{Author biography}

Kavitha Mohankumar, Junior Resident

Abhay Amrit Lune, Professor

Rakesh Goud, Associate Professor

Chaitali Chandrashekhar Desai, Junior Resident

Namratha Judith Cardoza, Junior Resident

Cite this article: Mohankumar K, Lune AA, Goud R, Desai CC, Cardoza NJ. Study of prevalence of raised IOP in post cataract patients following topical steroid usage. Indian J Clin Exp Ophthalmol 2021;7(2):436-441. 